\title{
LES DÉRIVÉS DU NOM PROPRE TRUMP DANS LE CONTEXTE DE LA CRISE SANITAIRE : APPROCHE SÉMANTIQUE ET MORPHOLOGIQUE
}

Tout événement politique ou social s'accompagne de créations lexicales et la crise sanitaire du coronavirus n'y fait pas exception. Si l'avènement de lexèmes composés comme " geste-barrière » (souvent au pluriel d'ailleurs) était prévisible - tant l'actualité reste focalisée sur le sujet - on aurait pensé observer un net recul des néologismes intéressant directement notre recherche, à savoir les dérivés du nom propre «Trump ». Mais c'était sans compter sur l'arrivée en force du coronavirus sur le territoire américain et l'omniprésence médiatique du président des Etats-Unis. Cette combinaison a abouti à la création de nombreux néologismes qui consignent les traces d'un produit de la crise du coronavirus : un parallélisme détonnant entre le virus et une politique donnée.

D’une manière générale, la dérivation des noms propres étant assez fréquente, surtout dans les textes journalistiques, nous sommes consciente que les dérivés du nom propre Trump s'ajoutent à quantité d'autres créations lexicales en lien avec la politique, notamment d'autres noms de présidents comme par exemple « reaganisme » ou " mittérandisme ». Il serait sans aucun doute fructueux de comparer ces dérivés et de les replacer dans un contexte plus large, toutefois, le format d'un article ne permet pas une analyse d'une telle ampleur. Malgré cela, nous estimons que Donald Trump, demeurant en ce moment au centre de l'intérêt des médias, se montre un choix pertinent pour notre recherche. Non seulement parce qu'il constitue un sujet d'actualité mais aussi pour la manière dont les informations circulent en cette époque de plus en plus numérique : les réseaux sociaux et Internet, plus favorables à la création néologique que la presse imprimée, sont devenus l'alpha et l'oméga du journalisme et de la transmission de l'actualité.

Dans cet article, nous procéderons en deux temps : d'abord nous reviendrons sur des dérivés du nom propre «Trump » qui, sans être très anciens, le sont néanmoins plus que ceux que nous analyserons ensuite dans la seconde partie, à savoir les néologismes qui mettent en œuvre le parallélisme évoqué plus haut. 


\section{2 « TRUMPIEN », « TRUMPISME » : DES DÉRIVÉS DÉJÀ INTÉGRÉS}

Comme nous l'avons laissé entendre, les néologismes actuels viennent accentuer un phénomène actif depuis quelques années : les dérivés du nom propre Trump créés entre 2016-2020. Actuellement, la liste des néologismes, repérés majoritairement par une recherche en ligne (moteurs de recherche et relevé manuel quotidien dans la presse), compte plus de 90 lexèmes et, la crise du coronavirus en est la preuve, cette liste ne cesse de s'accroître. Pourtant, plus que les chiffres, quoique dignes de l'attention, ce sont les particularités sur les plans morphologique et sémantique que l'ensemble des dérivés présente qui méritent d'être soulignées.

Ces deux pistes, morphologique et sémantique, sont les piliers de notre recherche ce qui correspond, sous l'optique saussurienne, à l'idée du double groupement. Sur l'axe syntagmatique, nous nous intéressons à deux séries paradigmatiques : d'un côté à des séries d'affixes (majoritairement des suffixes) soudés au nom propre Trump et de l'autre côté sur des séries de bases dérivatives de noms propres interchangeables avec «Trump » en observant les liens entre eux. Le schéma suivant illustre ces deux groupements sur l'exemple de l'adjectif « trumpien»:

\begin{tabular}{lll} 
& $\overline{\text { Trump-ien }}$ & \\
\cline { 2 - 2 } Macron-ien & Trump-isme \\
Gaull-ien & Trump-iste \\
Clinton-ien & & Trump-erie
\end{tabular}

Ce groupement qui s'applique également sur la deuxième partie consacrée au parallélisme viral accentue l'importance de l'opposition pour la délimitation du contenu sémantique.

En effet, la créativité néologique journalistique se manifeste principalement par la richesse et variété morphologique des lexèmes liés au nom du président américain : de nombreux procédés de formation produisent des dérivés affixaux (p. ex. anti-Trump, trumpiste, untrumpable), des mots composés (p. ex. trumpéconomie), des mots-valises (p.ex. trumpocalypse, trumputin), voire des défigements (p. ex. trumpement de terre, SchTrump). Leur apparition, pourtant, pourrait ne paraître que guère surprenante : la théorie saussurienne sur l'analogie expose en détail que toute création résulte d'une comparaison préalable : « Toute création doit être précédée d'une comparaison inconsciente des matériaux déposés dans le trésor de la langue où les formes génératrices sont rangées selon leurs rapports syntagmatiques et associatifs. » (Saussure, 1917 : 227). Néanmoins, d'après Saussure, ces créations sont des œuvres occasionnelles qui appartiennent à la parole et non à la langue. Si la majorité de ces créations tombent dans l'oubli par leur caractère occasionnel lié à l'actualité, comme c'est le cas de nombreux dérivés des patronymes politiques (Martincová, 1983 : 
23 ; Colin, 2003 : 418), il convient de mettre en relief ce qui est peut-être moins apparent au premier aspect : le resserrement sémantique de certains de ces néologismes.

Les noms propres étant dépouillés de sens dans la conception traditionnelle où un nom propre se limite au sème /être appelé/, l'anticipation des adjectifs purement relationnels comme résultats de dérivation par l'ajout d'un suffixe non-marqué (Lignon, 2002 : 2) serait logique. Pourtant, certains exemples (dont notre lexème « trumpien ») témoignent du contraire et leur emploi dans les textes dépasse clairement la catégorie des adjectifs relationnels. Comment ce resserrement se manifeste-il ? Illustrons-le à l'aide du contexte qui contient souvent toute une définition de cet adjectif :

Une caractéristique unique du style trumpien est sa capacité de s'adresser directement à une clientèle électorale particulière, sa «base », qui réside partout où le Parti républicain est le mieux enraciné, particulièrement dans les régions du centre et du sud des États-Unis. ${ }^{1}$

Dans d'autres extraits, le passage possible du «trumpien » à une normalisation des emplois comme adjectif qualificatif s'annonce par la morphologie : la gradabilité étant une des caractéristiques essentielles des qualificatifs (Fradin, 2008 :2), elle indique une adjonction de sèmes supplémentaires :

Donald Trump est resté plus trumpien que jamais. Cela donne une idée assez claire de ce que sera sa stratégie jusqu'en 2020 : continuer sur sa lancée. ${ }^{2}$

Ce qui mérite également d'être mis en relief, c'est l'emploi de l'adjectif « trumpien » comme désignation d'un comportement politique pourtant difficilement saisissable. Il semblerait qu'il serve de critère d'évaluation et de catégorisation des personnages politiques ou de leurs propos :

Jair Bolsonaro serait-il plus trumpien que Trump lui-même? Le président brésilien d'extrême droite est connu, comme son homologue de la Maison-Blanche, pour ses coups de sang et sa relation conflictuelle avec la presse. ${ }^{3}$

Lorsque M. Trump a augmenté les droits de douane sur les produits chinois au début du mois dernier, M. Schumer a tweeté : "Tenez bon avec la Chine,

1 https://quebec.huffingtonpost.ca/michel-fortmann/la-communication-politique-a-l-ere-trumpienne_a_23488805/?guccounter=1\&guce_referrer=aHR0cHM6Ly93d3cuZ29vZ2x1LmNvbS8\&guce_referrer_sig=AQAAAMaQv85PQuePDK73Buss7I1fBMi_pmOzk0vMWtEm2GXMbOMx_38Yd1vKrY0n1FraM2qVqaYze6DoRn4kEqz2mCBKNf1zXpu9f8Tv9uLgfIsJDJXuO0aGzlVtiyMhj65Db12nba0kQEH-5TJZJi01y4cotwbBcMNaDLTS5hNG46UK

$2 \mathrm{https} / /$ www.europe1.fr/international/aux-etats-unis-la-presidentielle-2020-commence-maintenant-3797156

3 https://www.parismatch.com/Actu/International/La-folle-semaine-du-Bresil-de-Bolsonaro-1656438 
Président (Trump). Ne reculez pas. La force est le seul moyen de gagner avec la Chine. " M. Schumer semble parfois presque trumpien lorsqu'il parle des pratiques commerciales chinoises, les qualifiant de "prédatrices » et disant que Pékin est un «concurrent déloyal » qui « tord et enfreint les règles $»^{4}$

Le suffixe -ien s'avère donc particulièrement productif (les extraits ci-dessus ne sont qu'un échantillon très limité) en jonction au nom propre du président américain. La racine de ce phénomène est, à notre avis, double : c'est d'abord l'omniprésence de Donald Trump dans les médias et sa notoriété auprès de la population mais aussi les caractéristiques morphologiques du suffixe -ien. En effet, ce suffixe relationnel qui s'ajoute à des bases nominales en exprimant souvent l'appartenance ou l'origine (Lignon, $2000: 3$ ), devient facilement qualificatif (Leroy, $2005: 1$ ).

La présence d'autres courants et personnages politiques dans le contexte textuel des dérivés, comme c'est le cas de " trumpien », ne s'arrête pourtant pas là : la catégorie des substantifs en est également affectée. Nous pouvons l'observer particulièrement sur l'exemple du dérivé " trumpisme » dont la création n'a rien d'étonnant : le suffixe -isme s'ajoute souvent aux noms propres et avec facilité (Guiraud, 1968 : 62 ; Ducháček, 1976 : 42, Niklas-Salminen, $2015: 64)$. Le phénomène en n'est pas moins intéressant, au contraire. Depuis l'élection de Donald Trump, la définition de cette politique atypique ne cesse de se cristalliser dans la langue : le «trumpisme », quoiqu'il ne soit pas ancré officiellement dans le lexique français, constitue à présent un ensemble riche des définitions fortement marquées d'un point de vue axiologique : pour ne prendre que deux adjectifs des tentatives de définitions suivantes, relevons ci-après : «brutal » et « radicale ».

Le trumpisme, un retour brutal à des relations internationales fondées sur les rapports de force. ${ }^{5}$

Tout en s'inscrivant dans le mouvement de repli national que l'on peut observer dans de nombreux points du globe, le "Trumpisme ", entendu comme mouvement de réorientation potentiellement radicale des politiques économiques intérieure et extérieure des États-Unis sous l'impulsion de Donald Trump, présente des particularités importantes. ${ }^{6}$

Le «trumpisme », en tant que sémème, est d'ailleurs intéressant non seulement pour l'abondance des sèmes qui le délimitent et le cernent, mais aussi par les moyens dont les contours du sens de ce dérivé sont exprimés : des corrélats sémantiques à teneur superlative (cf. ci-après « paroxysme », « suprématie », puis « grave », « dictature ») et nettement

4 https://www.lopinion.fr/edition/wsj/etats-unis-republicains-democrates-main-dans-main-contre-chine-tech-189513

5 https://www.france24.com/fr/20190618-invite-jour-gerard-chaliand-trump-etats-unis-iran-affrontement-oman

6 https://cecmc.hypotheses.org/34012 
dysphorique « affection », « misogynie », « homophobie », « racisme », puis « danger », « fascisme », etc.) l'accompagnent régulièrement.

Le trumpisme est le paroxysme d'une affection latente caractéristique des dernières années dans nos sociétés occidentales. C'est la manifestation politique d'une lame de fond porteuse de nationalisme identitaire, de suprématie blanche, de misogynie machiste, d'homophobie, et de racisme. ${ }^{7}$

Le trumpisme et ce qu'il engendre constituent aujourd'hui un grave danger. Entre, d'une part, la démocratie bourgeoise habituelle et tous ses défauts et, d'autre part, le fascisme, il y a des formes intermédiaires, comme la dictature militaire. Il y a aussi toute une gamme de formes possibles dans ce que Marx appelait le bonapartisme. ${ }^{8}$

La richesse des dérivés en -isme comprise dans les contextes du « trumpisme » est étonnante : il est à la fois classé dans des cadres plus généraux tels que le néoconservatisme, le racisme ou le fascisme et il se met en opposition graduelle à d'autres courants dérivés des noms des personnages politiques importants, comme le «bonapartisme » ci-dessus. Malgré la quantité de ces courants et personnages, un homme politique apparaît dans les extraits de la presse française plus fréquemment que d'autres : Emmanuel Macron.

Cette différence de conception du trumpisme et du macronisme se traduit sur leurs visions du monde. Certes, elles sont assez proches. Pour l'un comme pour l'autre il y aurait un centre et des périphéries, le centre imposant son ordre et ne laissant d'autres choix aux périphéries que de se soumettre. Ce pendant ce qui les distingue, c'est que pour le trumpisme le centre serait réduit à l'Occident, plus précisément aux puissances capitalistes dominantes du monde occidental, celui-ci étant soumis à l'hégémonie américaine. Par contre, pour le macronisme "l'hégémonie occidentale est profondément remise en cause » et sill y a bien un centre celui-ci serait constitué de l'Occident et de quelques autres puissances devenues incontournables au premier chef, l'Inde et la Chine. ${ }^{9}$

Macron et Trump, deux réactions oligarchiques différenciées à la crise, sontils la preuve que le capitalisme s'accommode finalement aussi bien du chauvinisme économique et culturel que du progressisme? Du trumpisme et du macronisme, lequel est le stade suprême du capitalisme ? ${ }^{10}$

7 https://www.ifri.org/fr/espace-media/lifri-medias/etats-unis-2020-trumpisme-infaillible

8 https://alencontre.org/ameriques/americnord/usa/etats-unis-debat-quest-ce-que-le-trumpisme.html

9 https://blogs.mediapart.fr/hillel-roger/blog/110919/trumpisme-et-macronisme

10 https://www.reconstruire.org/kevin-victoire-le-stade-supreme-du-capitalisme-cest-le-macronisme/ 
Malgré la polarisation évidente de ces deux idéologies dans ces extraits, il convient d'évoquer que certains auteurs y trouvent également des points d'intersection :

Macronisme et trumpisme, deux idéologies parentes ? ${ }^{11}$

L'arrivée au pouvoir d'Emmanuel Macron et de Donald Trump a consacré un nouveau type d'homme politique, qui a tous les traits du dirigeant économique et dont le style fait école avant même que ses recettes aient été véritablement testées (" macronisme », " trumpisme »). ${ }^{12}$

L'objectif de cette section étant de préparer le terrain de la suivante, nous ne pousserons pas plus avant ces observations qui concernent des faits moins en lien avec l'actualité centrale de ce début d'année 2020.

\section{3}

\section{LE TRUMPISME À L’EPRÉUVE DE LA PADÉMIE}

La connexion supposée entre macronisme et trumpisme se trouve réactivée dans la situation actuelle de crise sanitaire mondiale en assimilant le message politique à une maladie, nettement motivé par l'idée de la propagation du virus dévastateur. Une politique particulière, en l'occurrence trumpienne ou macronienne, est donc pourvue du sème /contagieux/ contenu dans les prédicats « infecter» et « être atteint». Aussi, remarquons que le -virus est intégré dans une construction active comme passive. Mais aussi et surtout ses cooccurrents 'ADN', 'famille' et deux fois 'espèces' qui appellent le paradigme « viral » décliné par la suite.

Les Macronavirus sont des petits virus à ADN double brin de la famille des Patronaviridae connus pour infecter l'homme. Parmi les espèces virales proches on trouve des espèces éteintes, comme les Regeanovirus ou Thatcherovirus, et des espèces contemporaines comme les Trudeauvirus, Salvinivirus, Bolsonarovirus, ou les Trumpvirus. ${ }^{13}$

Dans ce passage, 'double brin' semble détourner 'double hélice', substitution qu'il est difficile d'interpréter clairement. Alors qu'on aurait pu s'attendre à trouver, s'agissant de virus, " $\mathrm{ARN}$ », on a bien ADN, ce qui ne laisse planer aucun doute sur l'obédience humaine du virus. Cet aspect est d'ailleurs confirmé par l'onglet déroulé qui oppose vieux virus ( «espèces éteintes ») et nouveaux (« espèces contemporaines ») : le menu déroulant ne dévoile que des noms propres (ce qui explique « Patronaviridae », tous des hommes

\footnotetext{
11 https://www.cairn.info/revue-savoir-agir-2018-2-page-5.htm?contenu=resume\#

12 https://www.bilan.ch/opinions/souffle_liberal_ou_souffle

13 http://macronavirus.ouvaton.org/
} 
ou femmes politiques présidents ou premiers ministres : Reagan et Thatcher pour la première catégorie et Trudeau, Salvini, Bolsonaro, et Trump pour la seconde.

... les deux tiers des Français sont atteints par le macronvirus : ils n'ont pas confiance dans Emmanuel Macron et sont convaincus que les autorités leur mentent; compte tenu de ce qui précède, il est bien difficile de ne pas penser comme eux. ${ }^{14}$

Les -virus politiques sont ainsi mis en parallèle avec le « vrai » coronavirus à travers l'idée de /diffusion/ : le coronavirus sert donc de base à une sorte d'allégorie :

Malgré cela, le New York Times rebaptise le Coronavirus en " Trumpvirus » (sic !) et les " démocrates », qui se réjouissent ouvertement du fléau, critiquent à tout va, contre faits et statistiques. ${ }^{15}$

Les deux menaces peuvent également être coordonnées, et ainsi placées comme deux sous-catégories de virus :

Combattre le coronavirus et le macronavirus, en « même temps $»^{16}$

Dans d'autres extraits, les dérivés sont plutôt un contrepoids du danger du coronavirus. Les deux dangers sont comparés ou même mis en opposition : ainsi, le coronavirus est non plus un pouvoir maléfique mais un porteur d'espoir de guérison de l'autre comme dans le deuxième extrait ci-dessous :

Le trumpvirus est plus dangereux que le coronavirus ${ }^{17}$

"Le coronavirus peut-il guérir l'Amérique face au Trumpavirus ? " s'interroge Philippe Labro avant de conclure : "Novembre est loin... mais la paille a brisé le dos du chameau. $»^{18}$

Il est intéressant de noter que les deux néologismes composés présentent deux formes de suffixes qui se comportent comme des allomorphes sans conditionnement particulier (ni phonique ni morphématique) et le choix de l'un ou l'autre revient au locuteur : une version raccourcie, « trumpvirus » ou « macronvirus » contre « trumpavirus » et « macronavirus »

14 http://neuro.over-blog.com/2020/03/le-scandale-de-creil-et-le-macronvirus.html

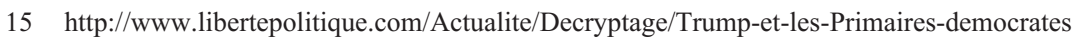

$16 \mathrm{https} / / / \mathrm{blogs} . m e d i a p a r t . f r / p a s c a l-s a n t o n i / b l o g / 100320 /$ combattre-le-coronavirus-et-le-macronavirus-en-meme-temps

17 https://www.brain-magazine.fr/article/page-president/59887-Le-trumpvirus-est-plus-dangereux-que-le-coronavirus

18 https://www.lepoint.fr/invites-du-point/philippe-labro/philippe-labro-le-coronavirus-peut-il-guerir-l-amerique-dutrumpavirus-26-04-2020-2372964_1444.php\# 
qui maintiennent le $-a$ - entre les deux éléments composants. Son insertion pourrait être justifiée soit par la tendance à imiter le lexème d'origine, le coronavirus, soit par des raisons phonologiques, le $-a$ - facilitant la prononciation.

D’autres exemples sont dérivés de l'idée de pandémie, terme pourvu de corrélats habituels comme 'foyer', 'infection', 'sanitare'. Pourtant, la tension politique qui s'élève au sein de la crise y ressort encore plus clairement : dans le cas de « Trumpandémie », le néologisme exprime une sorte de gradation de la controverse entre les États-Unis et la Chine qui l'emploie pour mettre en relief sa conviction au sujet de la provenance du virus :

Sortirons-nous de cette macropandémie décimés, asservis, misérables? La première mesure sanitaire, urgente, c'est de libérer nos esprits de l'emprise de ce foyer d'infection moral qu'est le pouvoir actuel. ${ }^{19}$

Les États-Unis comptent sur les mensonges et le racisme au lieu de la science et de mesures efficaces pour freiner \#COVID19. Jeter un coup d'œil@thedailybeast sur les tactiques vilaines de la Maison Blanche pour détourner les critiques sur \#Trumpandémie. ${ }^{20}$

Il demande enfin des explications sur les accusations de délit d'initiés nées après la vente d'actions de la part de sénateurs américains, survenue avant la chute de la Bourse liée à l'épidémie. Le même jour, l'ambassade chinoise en France parlait même de "Trumpandémie ${ }^{21}$

Enfin, souvent plus éloquentes que les textes, les caricatures incarnent l'idée de cette fusion politico-sanitaire. Certains néologismes ainsi ne sont donc pas intégrés à des textes mais accompagnent seulement des dessins, ce qui empêche de les analyser plus avant :

18 mars 2020 - Trumpavirus... Coronatrump...

Ce rapport entre deux courants décrits comme à la fois voisins et contradictoires qui, de plus, interagissent avec les sujets d'actualité, ne met en lumière qu'une partie

19 https://www.egaliteetreconciliation.fr/Macronovirus-58514.html?_cf_chl_captcha_tk_=0761bd3a211029a7b74184abaf770caf33074388-1587732102-0-ATR-eC8th Y PXVY4svOc2ehZKDtuqFE4kuhldfCjFYQ1xHg0Ne8QeY3ukY6IphxMNFoq1XDw3wjtYqXj_2Mnm6OK5Q-q4SE0gKeQiolT0fIKS6XWVP-U5wc09itDrYA9dI220DpwUUc1N_agorTt0VI81NRaBIw8bBFYL-Ut4xeREb4oVD8fTYq0jIiiV91H5a4Z4WGRXewkfR1b97PcHPFOEeBb_7kw4F8RHko30P1BcHVemvvxAdEGwKoFY2fP3L391eHEy90LxHXQP9xyRgNmpDXZFLTdNTmČcasCd_91BtDKfBJ67vE1SSR9Wu31TVNgzr1gvvtkjXItKGIx3kZe6AN59UZXTDuJqKNNHRNCEn0p6RmCoiMwgmhvQpyGCO48qhzxEPHvztMq7XxJY8Dn0ZoS0UqNxYLUT-kkPukTnxRcUK8QyLMCk3vM-rK5rYr2jYdftmpNq16r8IyHMy5PaCBRTI_9a1uoknbIeZFxcBRK-6AwvvjgNAKkKJszOEGKqfvkeNi_eDVp0nHrjQ

20 https://witter.com/AmbassadeChine/status/1242114229739622400

$21 \mathrm{https} / /$ www.parismatch.com/Actu/Sante/Quand-la-diplomatie-chinoise-fait-la-promotion-de-theories-du-complot-sur-le-coronavirus-1679965 
d'un paradigme beaucoup plus vaste : une quantité de composés et dérivés font leur apparition dans la presse, sans que la base ne soit nécessairement un nom propre, tels que « corbeaunavirus » :

Corbeaunavirus : Réjouissance du confinement : il a fallu moins d'un mois pour que certains Français renouent avec une sympathique tradition vieille de plus de quatre-vingts ans. C'est "le retour des corbeaux » s'écrie Le Monde (11/4). Oui, ces corbacs, qui sous l'Occupation, espionnaient et balançaient leur voisin sous le noble prétexte de préserver la société. ${ }^{22}$

Ceci exclut donc la spécialisation de l'application du pseudo suffixe «-(n)(a)virus » à la catégorie du nom propre.

\section{$4 \quad$ CONCLUSION}

Il est bien délicat de prédire le devenir de ces néologismes, presque autant que l'évolution de la pandémie. Mais plusieurs faits sont à retenir ici : au niveau morphologique, le statut de suffixe provisoirement acquis par «-(n)(a)virus », sur la base du modèle d'actualité « coronavirus », mot inconnu ou presque il y a quelques mois. Et d'un point de vue sémantique, la projection des sèmes qui le définissent, par suffixation, sur les noms propres qui servent de base au dérivé, en l'occurrence « Trump » pour ce qui nous concerne. Ce courant politique, dont la définition se concrétise en fonction des actes de Donald Trump et ainsi reste toujours assez complexe, est, de plus, comparée ou opposée à d'autres courants comme le «macronisme », même dans le contexte du coronavirus. À ces deux idéologies souvent comparées ou opposées s'associent des sèmes /diffusion/, /contagieux/ ou /danger/ et ainsi les dérivés passent au niveau abstrait pour décrire un parallèle entre le coronavirus et la contamination par une idéologie.

S'ils restent des occasionnalismes après la crise sanitaire, ils n'auront pas seulement incarné la créativité lexicale qui accompagne une époque particulière mais ils auront reflété un parallélisme conceptuel politique-virus, qui, sans être inédit, est à la fois remarquable et observable en temps réel.

\section{BIBLIOGRAPHIE}

BOULANGER, Jean-Claude/Monique C. CORMIER (2001) Le nom propre dans l'espace dictionnairique général: Études de métalexicographie [en ligne]. Tübingen : Max

22 Le Canard Enchaîné du 15 avril 2020. 
Niemeyer Verlag, https://books.google.cz/books?id=3VPoBQAAQBAJ\&pg=PA13 $5 \& 1 \mathrm{pg}=\mathrm{PA} 135 \& \mathrm{dq}=\mathrm{d} \% \mathrm{C} 3 \% \mathrm{~A} 9$ riv $\% \mathrm{C} 3 \% \mathrm{~A} 9+\mathrm{du}+$ nom + propre $\&$ source $=$ bl\&ots $=\mathrm{G}-$ M115fm8-n\&sig=ACfU3U3VbNA9vyhGTQi8OTafiib_oUXK7A\&hl=cs\&$\mathrm{sa}=\mathrm{X} \& \mathrm{ved}=2 \mathrm{ahUKEwiFi8WIzI}$ _pAhVZ5eAKHXJ2D3Q4ChDoATAFegQI$\mathrm{CRAB} \# \mathrm{v}=$ onepage $\& \mathrm{q}=\mathrm{d} \% \mathrm{C} 3 \% \mathrm{~A} 9 \mathrm{riv} \% \mathrm{C} 3 \% \mathrm{~A} \% 20 \mathrm{du} \% 20$ nom $\% 20$ propre $\& \mathrm{f}=-$ false, consulté le 30 avril 2020.

CUSIMANO, Christophe (2015) Kafka-like et kafkaïen : approche morphologique et sémantique des dérivés du nom d'auteur Kafka. La Linguistique [en ligne] 51, 269290. https://www.jstor.org/stable/24886389, consulté le 5 mai 2020.

DUCHÁČEK, Otto (1976) Grammaire du français contemporain. Bratislava: Slovenské pedagogické nakladatel'stvo.

JACQUET-PFAU, Christine/Jean-François SABLAYROLLES (2016) La fabrique des mots français: colloque de Cerisy. Limoges, France : Lambert-Lucas.

JONASSON, Kerstin (2005) La modification du nom propre dans une perspective contrastive. La langue française 146 [en ligne], 67-83. https://www.cairn.info/revue-langue-francaise-2005-2-page-67.htm, consulté le 11 mai 2020.

LEROY, Sarah (2005) Les dérivés de noms propres dans le TLFi : quelles bases pour quels sens ? Corela [en ligne] HS-1. https://journals.openedition.org/corela/1146\#quotation, consulté le 21 avril 2020.

LIGNON, Stéphanie (2002) L'adjectif en -ien comme révélateur de phénomènes de concurrence. BULAG - Bulletin de linguistique appliquée et générale [en ligne] 27, 135-150. http://w3.erss.univ-tlse2.fr/textes/publications/CE/Lignon.pdf, consulté le 21 avril 2020.

MARTINCOVÁ, Olga (1972) K problematice lexikálních neologismů. Slovo a slovesnost 33 [en ligne], 283-293. http://sas.ujc.cas.cz/archiv.php?art=219, consulte le 21 avril 2020 .

MOLINO, Jean (1982) Le nom propre dans la langue. Langages 66 [en ligne], 5-20. http://www.persee.fr/web/revues/home/prescript/article/lgge_0458726x_1982 num_16_66_1123, consulte le 21 avril 2020.

MORTUREUX, Marie-Françoise (2002) Ephémérité est-il français ? Linx 12 [en ligne], 195-202. https://journals.openedition.org/linx/1304?lang=fr\#tocto2n2, consulté le 23 octobre 2020.

NIKLAS-SALMINEN, Aino (2015) La lexicologie. Paris : Armand Colin.

RASTIER, François (2012) Néologismes et néonazisme. Sur le diagnostic d'Anders Breivik. Cités 50 [en ligne], 13-17. https://www.cairn.info/revue-cites-2012-2page-13.htm, consulté le 5 mai 2020.

SABLAYROLLES, Jean-François (2000) La néologie en français contemporain: examen du concept et analyse de productions néologiques récentes. Paris : H. Champion, 2000.

SAUSSURE, Ferdinand de (1971) Cours de linguistique générale (1916). Paris : Pyot. 
POVZETEK

\section{TVORJENKE IZ LASTNEGA IMENA TRUMP V KONTEKSTU ZDRAVSTVENE KRI- ZE: POMENOSLOVNI IN OBLIKOSLOVNI VIDIK}

$\mathrm{V}$ prispevku se osredotočamo na oblikoslovno in pomenoslovno analizo neologizmov, tvorjenih iz lastnega imena »Trump«. Tvorjenke v prispevku predstavljamo z oblikoslovnega vidika, hkrati pa s pomenskega vidika analiziramo njihovo rabo, ki je prikazana $v$ kratkih odlomkih iz časopisnih člankov. Pri tem se osredotočamo predvsem na aktivnost pomenskih sestavin predstavljenih neologizmov.

Prispevek je razdeljen na dva dela, v katerih so predstavljeni primeri omenjenega novega besedotvornega pojava. Najprej je obravnavan francoski pridevnik »trumpien«, katerega pripona -ien tvori tako vrstne kot kakovostne pridevnike. Tvorjenke iz lastnega imena ameriškega predsednika vsebujejo pomenske sestavine, ki prispevajo tudi k pomenu tvorjenke »trumpisme«. Slednjo predstavljamo v drugem delu prispevka. Opredelitev njenega pomena omogočata velika pogostnost rabe in primerjava $\mathrm{z}$ drugimi izpeljankami s pripono -isme, med katerimi je najpogosteje rabljena beseda »macronisme«.

Različna besedila še vedno ustvarjajo nove pomenske povezave med besedama »trumpisme« in »macronisme«. To je postalo še očitnejše med koronavirusno krizo. Pričakovali bi, da bo tako pereča tema, kot je svetovna pandemija, v medijih povsem zasenčila politično dogajanje. Prav nasprotno pa je zdravstvena kriza spodbudila leksikalno kreativnost na tem področju, predvsem z rabo novih morfemov, povezanih s pomenskim poljem pandemije, kot so -virus, -pandémie ali corona-. Tako je širjenje koronavirusne bolezni postalo metafora za širjenje določene politične ideologije - v našem primeru ideologij Donalda Trumpa in Emmanuela Macrona.

Ključne besede: lastno ime, oblikoslovje, pomenoslovje, besedotvorje, koronavirus, Donald Trump

\section{ABSTRACT}

\section{DERIVATIVES FROM THE PROPER NOUN "TRUMP" IN THE CONTEXT OF THE HEALTH CRISIS: A SEMANTIC AND MORPHOLOGICAL APPROACH}

The article focuses on neologisms derived from the proper noun "Trump" from the morphological and semantic point of view. Our morphological observation is accompanied by short journalistic extracts with comments; the emphasis is put on neologisms' semic activity.

Organised in two parts, the article points out some examples of this recent phenomenon. First of all, we are concerned with the French adjective "trumpien" whose suffix -ien produces not only relational but also qualifying adjectives. In other words, supplementary semes join the derivative of the American President's proper noun and in this way they specify the definition of "trumpisme", the second example described in the article. Numerous occurrences of the derivative allow 
us to delimit the semantic content of this neologism, besides other things, by putting it in opposition or in parallel other-ismes, with "macronisme" arguably the most common one.

The link established between the "trumpisme" and the "macronisme" is still being actualised, and it has been intensified during the coronavirus crisis. We could suppose that an acute topic such as the world pandemic would not allow any space for politics in the media. However, the medical crisis actually boosted the lexical creativity in this field using new elements connected to the pandemic like -virus, -pandémie or corona-. Thus, spreading of the coronavirus becomes a metaphor of spreading of a specific political ideology, in this case Donald Trump's and Emmanuel Macron's.

Keywords: proper noun, morphology, semantics, derivation, coronavirus, Donald Trump

\section{RÉSUMÉ}

L'article se penche sur les néologismes dérivés du nom propre « Trump » d'un point de vue morphologique et sémantique. Nos observations morphologiques sont accompagnées de courts extraits journalistiques commentés avec un accent mis sur l'activité sémique des néologismes.

Organisé en deux parties, l'article relève quelques exemples de ce phénomène récent : d'abord, nous nous focalisons sur l'adjectif « trumpien » dont le suffixe très productif -ien forme non seulement les adjectifs relationnels mais également qualificatifs. En d'autres termes, des sèmes supplémentaires s'adjoignent au dérivé du nom propre du président américain et ainsi cisèlent la définition du « trumpisme », le deuxième exemple décrit dans l'article. Les nombreuses occurrences du dérivé permettent délimiter le contenu sémantique de ce néologisme, entre autres, par l'opposition ou mise en parallèle avec d'autres -ismes dont « macronisme » est sans doute le plus fréquent.

Ce lien établi entre le «trumpisme » et le " macronisme » ne cesse d'être actualisé en contexte : il s'intensifie même en cette période de crise du coronavirus. Nous pourrions supposer qu'un sujet aussi brûlant qu'une pandémie mondiale ne laisse pas de place pour la politique dans les médias : au contraire, la crise sanitaire a plutôt augmenté la création lexicale dans ce domaine en employant des éléments liés à la pandémie, tels que -virus, -pandémie ou corona-. L'expansion du coronavirus ainsi devient une métaphore de l'expansion d'une idéologie politique spécifique, en l'occurrence la politique de Donald Trump mise en opposition (ou en parallèle) avec l'idéologie supposée d'Emmanuel Macron.

Mots-clés : nom propre, morphologie, sémantique, dérivation, coronavirus, Donald Trump 